\title{
Norbert Lechner
}

\section{Marktgesellschaift und die Verånderung von Politikmustern}

\section{Die mögliche Demokratie}

Gerade in dem Moment als die demokratische Ordnung ein so hohes Maß an Anerkennung erlangt hat, wie noch nie zuvor, modifiziert eine radikale Veränderung ihres Kontextes den Umfang und die Bedeutung von Demokratie. Dies macht die Übergänge zu demokratischen Systemen noch komplexer. Demokratie ist nicht länger eine im voraus bestimmte fertige Konzeption, sie gewinnt vielmehr den Charakter einer historischen Bewegung zurück: Die selbstbestimmte Herstellung von Formen der Gemeinschaft für die Gemeinschaft als ein bewußt überlegter und kontinuierlicher Prozess (Castoriadis 1994). Wir sind Zeugen des Entstehens einer »neuen Demokratie «, die sich von den existierenden Formen, seien sie nun alt oder jung, unterscheidet. Diese Transformation ist um so schwerer wahrzunehmen, als sich die Akteure und die demokratischen Institutionen anscheinend nicht verändert haben. Doch diese formale Kontinuität verdeckt die Entstehung neuer Bedeutungen. Daraus folgt, daß die demokratische Frage vielleicht nichts anderes ist als die Suche nach der möglichen Demokratie. In welcher Weise beeinflussen die großen Transformationen, die im Gange sind, die Bedingungen der Möglichkeit von Demokratie?

Obwohl ich mich nur auf die demokratische Frage in Lateinamerika beziehe, haben die veränderten Politikmuster eine größere Reichweite, da sie auf globale Veränderungen reagieren, insbesondere auch auf ein neues »kulturelles Klima«. Konkrete Fallstudien können nur informativ sein, wenn wir gleichzeitig die Megatendenzen berücksichtigen, die sowohl die Form, wie Politik gemacht wird, als auch unser Bild von Politik bestimmen. In Lateinamerika, wie auch in anderen Regionen, wird die demokratische Frage vom Ende eines historischen Zyklus' und der integralen Reorganisation sozialer Zusammenhänge bestimmt (Calderon, Dos Santos 1991). Die rigorosen »Strukturanpassungen « haben die für die lateinamerikanischen Gesellschaften so charakteristische Staatszentriertheit (Cavarozzi 1991) drastisch verändert: Die Dynamik der sozialen Entwicklung verschob sich 
vom Staat auf den Markt. Da diese Restrukturierung weltweit stattfindet, besitzt sie unabhängig von den Besonderheiten der einzelnen Länder eine ungeheure Kraft. Der Kollaps der kommunistischen Staaten verleiht der Globalisierung kapitalistischer Marktwirtschaft eine höhere Weihe und legt gleichzeitig deren innere Spannungen bloß. Diese »Revolution« ist nicht auf die ökonomische und politische Weltkarte beschränkt; sie umfasst auch und vor allem unsere intellektuellen Koordinatensysteme. Wir verlieren die topoi der uns vertrauten Landschaft und entwickeln eine neue Vision der Welt und des Lebens. Ähnlich wie in den 20er und 30er Jahren, befinden wir uns jetzt am Ende des Jahrhunderts in einer Periode des Wandels, in der viele gebräuchliche Kategorien obsolet werden, wir aber noch nicht wissen, wie wir die neuen Tendenzen bezeichnen sollen.

\section{Modernisierung und Modernität}

Die Globalisierung der ökonomischen und finanziellen Prozesse, der technologische Wandel und der Kollaps der sozialistischen Länder lassen Entwicklungsmodelle, die lediglich die inneren Bedingungen eines Landes berücksichtigen, nicht mehr zu. Dadurch wird eine neue Phase der Modernisierung in Gang gesetzt, die sich von den Strategien der 70er Jahre unterscheidet. 1975 begannen Chile und andere lateinamerikanische Länder mit neoliberalen Strukturanpassungsprogrammen: Die öffentlichen Ausgaben wurden gekürzt, die Märkte wurden dereguliert und für ausländische Anbieter geöffnet und Staatsunternehmen wurden privatisiert. Die hohen sozialen Kosten dieser Maßnahmen sind bekannt; zu Beginn der 90er Jahre konnten 46\% der lateinamerikanischen Bevölkerung ihre Grundbedürfnisse nicht befriedigen und 22\% lebten in extremer Armut. Doch unerträglicher und noch weniger zu rechtfertigen als die Armut selbst, ist die Existenz von Hütten neben Palästen. Lateinamerika ist diejenige Region der Welt mit der größten sozialen Ungleichheit. Es lohnt, sich ein paar Statistiken der Weltbank ins Gedächtnis zu rufen, wenn wir die Situation in diesen Ländern analysieren. Vergleicht man das Fünftel der Bevölkerung mit dem höchsten Einkommen mit dem ärmsten Fünftel, so ist die durchschnittliche Relation in OECD Ländern 6,1:1, und in Asien 7,3:1; in Lateinamerika ist die Relation 18,6:1. Während in einem Land wie Bangladesh das reichste Fünftel ein sieben mal höheres Einkommen hat als das ärmste, ist das Einkommen des reichsten Fünftels in Peru 32 mal und in Brasilien 33 mal höher als das des ärmsten Fünftels (Larrain 1993). Unter diesen Umständen ist es schwierig, das Minimum an sozialer Kohäsion zu sichern, das für eine Gesellschaft notwendig ist. Wird öko-nomische Entwicklung nicht als Voraussetzung einer demokratischen 
Entwicklung betrachtet, so muß man fragen, welche Möglichkeiten eine demokratische Ordnung in einem solchen Kontext überhaupt besitzt.

Gleichzeitig können wir bei den größeren Ländern der Region zum ersten Mal von einer effektiven Marktgesellschaft sprechen. Das läßt sich, auch wenn man hier nicht generalisieren sollte, an der zentralen Rolle des Konsums illustrieren. In Chile besitzen zum Beispiel zwanzig Prozent der Bevölkerung eine Kreditkarte von einem der großen Kaufhäuser. Ratenkäufe sind nicht nur ein bevorzugter Mechanismus, soziale Disziplin durch unpolitische Mittel zu sichern, sie zeugen auch von einer gewissen Neigung der Menschen, auf die Stabilität oder Verbesserung ihrer Lebensbedingungen zu setzen. Zumindest in einigen lateinamerikanischen Ländern zeugt diese »Investition « in die bestehende Ordnung von der Dynamik, die die Marktgesellschaft und die »Vermarktwirtschaftlichung« der sozialen Beziehungen angenommen haben.

Bei der Diskussion des Neoliberalismus sind allerdings zwei Aspekte zu unterscheiden, die häufig vermischt werden. Zum einen wird der Ausdruck Neoliberalismus als Oberbegriff für unterschiedliche Strategien von struktureller Anpassung benutzt. Zum anderen meint Neoliberalismus im strengen Sinne die Rechtfertigung des Marktes als konstituierendes Prinzip einer integralen Reorganisation des sozialen Lebens. Während alle lateinamerikanischen Länder ihre Ökonomien auf die eine oder andere Weise an die neuen Modalitäten und Anforderungen des Weltmarktes anpassen, zeigen die Erfahrungen derselben Länder, daß die neoliberalen Bestrebungen, Politik durch den Markt als einziger Instanz sozialer Koordination und Regulation zu ersetzen, zum Scheitern verurteilt sind. Erfolgreiche Fälle ökonomischer Restrukturierung, wie Chile, bestätigen, daß der Markt allein eine soziale Ordnung weder schaffen noch aufrechterhalten kann. Die kapitalistische Marktwirtschaft ist in soziale und politische Institutionen und kulturelle Gewohnheiten eingebettet und ihr Funktionieren hängt ganz wesentlich von einer solcher Einbettung ab (Altvater 1991). Solange der institutionelle Kontext schwach ist, und eine konsistente politische Regulierung sozialer Prozesse nicht stattfindet, führen die ökonomischen Anpassungsstrategien zu sozialen Spannungen und gesellschaftlicher Fragmentierung. Aufgrund der verheerenden sozialen Folgen der neoliberalen Rezepte, gibt es in Lateinamerika eine breite Zustimmung zum neuen CEPAL-Ansatz einer sozial ausgewogenen, produktiven Transformation (CEPAL 1990). Dieses Konzept hat den Vorzug, den systemischen Charkter von Entwicklung hervorzuheben. Die lateinamerikanischen Erfahrungen zeigen, daß eine Weltmarktintegration, die auf nationaler Desintegration basiert, weder ökonomisch noch politisch lebensfähig ist. Die Modernisierung einiger Unternehmen, die dann auf dem Weltmarkt bestehen können, reicht eben 
nicht aus. Es ist vielmehr notwendig, daß die Gesellschaft als Ganzes am Entwicklungsprozess teilhat. Dazu muß den Konzentrations- und Exklusionstendenzen des Marktes gegengesteuert werden. Die zunehmende Segmentierung der Gesellschaft kann nicht mit rein ökonomischen Mitteln bekämpft werden, wie innovativ diese auch sein mögen. Dieser Zusammenhang kann anhand der Sozialpolitik verdeutlicht werden. Durch die Konzentration der Sozialausgaben auf die ärmsten Schichten wurden zwar wichtige Fortschritte erreicht, aber trotz solcher Bemühungen trägt der vorherrschende Ansatz wenig zur sozialen Integration bei. Zum einen geraten mit der ausschließlichen Betrachtung des Individuums als Wohlfahrtsempfänger kollektive Handlungsmöglichkeiten aus dem Blickfeld; zum anderen werden mit der einseitigen Betonung der Effizienz der Ausgaben andere wichtige Aspekte der Sozialintegration, z. B. auf der kulturell-symbolischen Ebene, ignoriert. In ihrem Kern bezieht sich Sozialpolitik nicht auf eine kollektive Ordnung; im Gegenteil, ihre lediglich auf einzelne Sektoren bezogene Herangehensweise widerspricht der ursprünglichen Idee der Solidarität, von der sie angeblich inspiriert wurde.

Lateinamerika leidet unter einer verspäteten Modernisierung, aber vor allem unter einem Mangel an Modernität. Eine Gesellschaft, die die soziale Ordnung nicht als eine Gemeinschaft von Bürgern konstituiert und reproduziert, erreicht allenfalls eine »Modernisierung ohne Modernität«. Das deutlichste Beispiel dafür ist Chile unter Pinochet. Hier fand eine grundlegende Reorganisation der Gesellschaft entsprechend einer Ziel-Mittel-Rationalität statt. Aber bis jetzt wurde diese einer instrumentellen Logik gehorchende Entfaltung von sozialen Beziehungen nicht von einer ethischpolitischen Reflexion begleitet, die die Normen und Werte, Sehnsüchte und Ängste berücksichtigt, die dem sozialen Handeln zugrunde liegen. In einem großen Teil der Region hat sich die Marktgesellschaft an den Rändern oder in Opposition zur politischen Gesellschaft und oft gewaltsam durchgesetzt. Die Modernisierungsprozesse folgen keiner bewußt herbeigeführten Orientierung und Zielsetzung, doch werden sie gleichzeitig im alltäglichen Verhalten der Menschen internalisiert. Der offensichtliche Selbstlauf und die faktische Normativität dieser neuen Dynamik provoziert Verzweiflung und Entfremdung. In früheren Jahren führte diese Situation zur Kritik des Kapitalismus im Namen des Sozialismus. Heute ist es für uns schwierig geworden, die Kluft zwischen der Logik des Marktes und dem politischkulturellen Bereich adäquat zu bezeichnen. Auf diese Weise entstehen verschiedene Formen mystischen Denkens als ein verstecktes Gesicht der Modernisierung.

Da die kapitalistische Marktgesellschaft eine Megatendenz unserer Epoche darstellt, kann sich Lateinamerika nicht von ihrer Dynamik abkoppeln, oh- 
ne Gefahr zu laufen, sich selbst von der historischen Entwicklung auszuschließen. Faktisch wird die Rationalität der Marktgesellschaft in den sozialen Beziehungen vorausgesetzt, während sie gleichzeitig auf der moralisch-normativen Ebene abgelehnt wird. Zwischen Modernisierung und Modernität existiert einen Spannung, die nicht einfach dadurch aufzulösen ist, daß der eine oder der andere Pol eliminiert wird. Im Gegenteil, wir müssen diese Spannung verdeutlichen und in jedem einzelnen Fall die involvierten Elemente kenntlich machen. Gerade davon handelt Demokratie, sofern sie nicht auf eine minimalistische Definition eines politischen Regimes reduziert wird, und sofern wir bereit sind, uns mit den Wechselbeziehungen von politischer Selbstbestimmung, sozioökonomischer Dynamik und symbolischer Repräsentation auseinanderzusetzen.

In den letzten Jahren ist die Entwicklung in Lateinamerika auf der Grundlage von zwei verschiedenen, meistens nicht miteinander verbundenen Ansätzen analysiert worden. Einige Arbeiten untersuchten die Prozesse des Übergangs zur Demokratie und betonten das strategische Handeln der Akteure sowie dic Rolle der Institutionen. Das Ziel der Übergangsprozesse wurde häufig als gegeben unterstellt, ohne daß berücksichtigt wurde, daß der veränderte sozio-ökonomische und kulturelle Kontext auch die Bedeutung von Demokratie neu definiert. Andere Arbeiten betonten dagegen die ökonomischen Anpassungsprozesse und hoben die produktiven, finanziellen und kommerziellen Transformationen sowie die an Bedeutung gewinnenden mikroökonomischen Veränderungen hervor. Erst in den letzten Jahren wurde auch im Rahmen dieser Studien nach den politischen und institutionellen Bedingungen der Durchführbarkeit von Reformen gefragt. Allerdings berücksichtigt keiner dieser beiden vorherrschenden Ansätze die langfristigen Veränderungen, denen die Politik selbst unterworfen ist (Cavarozzi 1994).

Deshalb ist es von einiger Bedeutung, daß die Inter-Amerikanische Entwicklungsbank (IADB) ihre Position kürzlich verändert hat und inzwischen die politischen Prozesse als entscheidende Faktoren für die Durchführbarkeit von ökonomischen Programmen betont (BID 1993). In Wirklichkeit sind die zentralen Entwicklungsprobleme in Lateinamerika eher politischer als ökonomischer Natur. Dabei handelt es sich aber nicht nur um die Frage demokratischer Regierungen; ich werde später darlegen, welche entscheidende Rolle die kulturelle Dimension von Politik in diesem Zusammenhang spielt. 


\section{Die Transformation der Politik}

Zunächst möchte ich die Aufmerksamkeit auf die Transformationen der institutionalisierten Politik lenken. Die schwierig zu klassifizierenden Veränderungen betreffen alte und neue Demokratien, rechte und linke Regierungen, Präsidialsysteme genauso wie parlamentarische. Daher ist der erste Schritt die Beschreibung einiger Restrukturierungsprozesse. Beginnen will ich mit der Neudefinition von Politik selbst.

Erstens, welche Bedeutung hat Politik heutzutage? Wenn es in einer modernen, säkularisierten Gesellschaft keine externen Legitimationsprinzipien mehr gibt und diese Gesellschaft deshalb gezwungen ist, Normen des sozialen Lebens aus sich selbst zu schöpfen, dann besteht das Primat der Politik darin, daß es sich bei ihr um die privilegierte Sphäre der Produktion und Reproduktion von das Gemeinschaftsleben regelnder Normativität handelt. Die Bedeutung moderner Politik besteht dann in der bewußten Gestaltung einer sozialen Ordnung.

In den letzten Jahren wurde das Fortschreiten von einer überkommenen Ordnung hin zu einer der Moderne entsprechenden, gestalteten Ordnung zunehmend durch die Idee einer sich selbst regulierenden Ordnung neu interpretiert. Damit wird die Zentralität von Politik als dem Scheitelpunkt der kollektiven Ordnungsleistungen in Frage gestellt. Faktisch tendiert Politik mehr und mehr dazu, vermittels Policy-Netzwerken zu operieren (Marin, Mayntz 1991), in denen sich auf formelle oder informelle Weise verschiedene soziale Akteure (Regierungsstellen, politische und soziale Gruppen) artikulieren, um Regelungen für bestimmte Fragen zu treffen. Das heißt, politische Entscheidungen werden tendenziell durch ausgehandelte Vereinbarungen ersetzt. Obwohl es im Einzelfall vielleicht keinen Unterschied macht, handelt es sich dabei aber um eine andere Art von politischer Produktivität. Politik wird deshalb gegenwärtig eher als ein Mechanismus der Koordination, denn als eine Sphäre von Führung und Leitung begriffen.

Zweitens, verweist uns die Schwächung von Steuerungskapazitäten auf eine Neubestimmung von Zeit. In einer moderne Gesellschaft wird die politische Macht als die bevorzugte Instanz betrachtet, welche in ein besseres »Morgen « führt. Die Zukunft erscheint nicht länger als Schicksal, sie wird vielmehr zu einem bewußt angestrebten Ziel. Die Fähigkeit politischer Führung wird aber auf die Behandlung von Kontigenzen reduziert, wenn sich nicht nur der Glaube an den Fortschritt in Nichts auflöst, sondern wenn auch der Begriff von Zukunft selbst verschwindet.

Die Allgegenwärtigkeit der Gegenwart nähert die Dynamik der Politik an die Logik des Marktes an. Auch der Markt hat einen Zukunftshorizont, 
aber nur in der Form von Gelegenheiten, Risiken und Spekulationen. Der Markt stellt Herausforderungen aber keine Ziele bereit. Dies setzt einen hohen Grad an Flexibilität voraus, die es ermöglicht, in angemessener Weise auf solche Herausforderungen zu reagieren. Diese Logik orientiert Politik auf eine Art von Management. Politik wird immer mehr als effektives Management aufgefaßt, das auf die gegebenen Herausforderungen reagiert, und nicht mehr als ein strategisches Handeln, das in Übereinstimmung mit von den Bürgern vorher definierten Zielen steht.

Drittens. Gleichzeitig verschieben sich die Grenzen des politischen Raumes. Der gegenwärtige Prozess der Globalisierung unterminiert die Kongruenz, die zwischen den Räumen von Politik, Ökonomie und Kultur, die alle eine einzige nationale Grenze besaßen, existiert hat. Die alte Spannung zwischen Demokratie und Kapitalismus drückt sich heute in dem Abstand aus, der die systemische Integration der Ökonomie auf der supranationalen Ebene von der politischen Integration trennt, die lediglich auf der Ebene des Nationalstaats stattfindet. Folglich setzen sich die externen Dynamiken in Ländern, die wie in Lateinamerika, eine nur schwache Sozialintegration aufweisen, direkt und unmittelbar durch und schwächen die prekären politischen »Filter « um so mehr.

Das demokratische Legitimationsdefizit bei Prozessen transnationaler Integration verweist darauf, daß das politische Feld in allen Gesellschaften neu organisiert wird. Wie wir in Lateinamerika beobachten können, wird die politische Sphäre in zunehmenden Maße von der ökonomischen in der Form »technischer Imperative« beschränkt. Makroökonomische Gleichgewichte repräsentieren ein neues normatives Prinzip, das der politischen Intervention rigorose Beschränkungen auferlegt. Angesichts früherer Erfahrungen erscheint es angebracht, daß die Politik nicht anstrebt, ökonomische Prozesse zu leiten, sondern die Logiken anderer Spären respektiert. Allerdings tendiert die institutionalisierte Politik heute dazu, Verantwortung in einem solchen Maße aufzugeben, daß man fragen muß, auf was oder wen Demokratie eigentlich noch reagiert.

Viertens. Die Neubestimmung der Grenzen von Politik verweist uns auf eine ganz wesentliche Veränderung: Die Ausweitung des Marktes auf nicht-ökonomische Sphären. Der Verweis auf den »politischen Markt« ist nicht länger eine bloße Analogie; Politik wird von der Dynamik des Marktes ergriffen. Dies ist Bestandteil eines komplexen kulturellen Prozesses, dessen offensichtlichste Erscheinung wahrscheinlich die Neudefinition des Öffentlichen und des Privaten ist.

Die Restrukturierung der öffentlichen Sphäre wurde ganz wesentlich durch die Privatisierung der Staatsunternehmen beeinflußt, die in Lateinamerika gegen Ende der 70er Jahre begann. Auch ohne die ökonomischen Konse- 
quenzen dieser Politik im Einzelnen zu diskutieren, ist heute offensichtlich, daß eine massive und wahllose Privatisierungspolitik nicht nur ökonomische Spannungen provoziert (wie jene, die 1982 in der Schuldenkrise explodierten), sondern auch die politische Sphäre, und damit eine fundamentale Såule der institutionellen Ordnung, aushöhlt. Indem ausschließlich die Wettbewerbsfähigkeit und Produktivität der Unternehmen, insbesondere des öffentlichen Dienstes, in Betracht gezogen wird, verschwindet die Vorstellung von »öffentlichen Gütern« und der Staatsbürger wird auf die Rolle des Konsumenten reduziert. Kurz: Durch die Schwächung der kollektiven Sphären führt die ökonomische Privatisierung auch zur Privatisierung des sozialen Zusammenhangs. Es entstehen neue soziale Formen, die auf individualistischen und utilaristischen Strategien aufbauen, und die für die Anpassung an marktvermittelte Wettbewerbsbeziehungen auch rational sind, die aber im Gegensatz zu jeder kollektiven Bindung und institutionellen Verantwortung stehen. Als Folge davon steigern diese neuen sozialen Beziehungen - unabhängig davon wie erfolgreich sie auf einer individuellen Ebene sein mögen - die Unsicherheiten und Risiken, so daß sie gleichzeitig die Ordnungsleistungen der Politik in Frage stellen.

Dieses neue Sozialverhalten ergreift auch das politische Handeln, das ebenfalls dahin tendiert, sich der Rationalität des Marktes zu unterwerfen. Wenn alle Werte auf austauschbare Güter zurückgehen und sich gegenseitige Anerkennung am merkantilen Kriterium des »do ut des « orientiert, dann verflüchtigt sich das politische Ethos von Verantwortung und Dienst an der Gemeinschaft, von Vertrauen und Loyalität. Die politische Klasse und die Staatsbürger sind dann nur noch in der Form eines kommerziellen Unternehmens miteinander verbunden.

\section{Die Transformation des Bolitischen}

Das Politische, d. h. die symbolische Repräsentation der kollektiven Ordnung, die die Grundsubstanz bildet, von der die institutionalisierte Politik lebt, bleibt bei der Analyse von Politik häufig ausgeklammert. Das »Politische« erlaubt keine substantielle Definition, mit der es ein für allemal bestimmt werden könnte; aber es handelt sich auch nicht um einen virtuellen Raum, der mit jedem Inhalt zu füllen ist. Das Politische verbindet die tägliche Erfahrung der Menschen mit der Gemeinschaft der Staatsbürger, und umschreibt die fließenden und sich verändernden Konstellationen von Elementen, die der bestehenden Ordnung entsprechen. Ignoriert man das »Politische«, so amputiert man das politische Phänomen und reduziert es auf seine institutionellen Ausdrucksformen, was nur seine Undurchsichtigkeit verstärkt. 
In den letzten Jahren wurde das Politische oft durch eine auffällige und allgemeine Eigenschaft charakterisiert: eine weitverbreitete, konfuse und schweigendes Unbehagen mit der Politik. In diesem Unbehagen scheint sich eine Abwehrhaltung gegen die oben beschriebenen Transformationen institutioneller Politik auszudrücken, gegen die sich, in Übereinstimmung mit bestimmten überlieferten Bildern von dem, was Politik ist und sein sollte, der Ärger richtet. Dieselben überlieferten Vorstellungen befinden sich aber gleichzeitig in einem Auflösungsprozess. Der neue Kontext verhindert, daß sich die alten Vorstellungen ohne weiteres reproduzieren können und macht Platz für einen Prozess der Neudefinition. Es erscheint angebracht, diese Veränderungsprozesse vor dem Hintergrund der herrschenden Malaise zu betrachten, da sich der einsetzende Rekonstruktionsprozess noch nicht zu einem neuen Konzept von Politik verdichtet hat.

In diesem Unbehagen uber die Politik erscheint der Widerspruch zwischen einer Politik, wie sie von den Bürgern wahrgenommen wird, und bestimmten überkommenen Vorstellungen. Vermutlich gehört die Auffassung von Politik als einer Instanz, die maximalen Schutz und Führung verkörpert, zu jenen grundlegenden, kollektiven Vorstellungen, die aufgrund ihrer Evidenz kaum expliziert werden, die aber ganz wesentlich zur politischen Kultur unserer Gesellschaften beitragen. Die Menschen erwarten, daß die Politik sie vor schicksalhaften Veränderungen schützt; das bedeutet nicht nur, daß ihre körperliche Unversehrtheit und ökonomische Sicherheit garantiert wird, sondern auch in einem ganz allgemeinen Sinne die Gewährleistung von Sicherheit. Von der Politik wird verlangt, die Entwicklung der Gesellschaft zu steuern und einen Blick auf die Zukunft zu ermöglichen, der die sozialen Prozesse verständlich und berechenbar werden läßt. Von dieser Perspektive aus gesehen provoziert die tatsächlich institutionalisierte Politik Mißtrauen. Der Verlust der Glaubwürdigkeit folgt meiner Meinung nach aus dem Eindruck, daß Politik die sozialen Prozesse nicht mehr kontrollieren kann. Die Dinge scheinen in einem Maße außer Kontrolle zu sein, daß die Bürger sich von einer ganzen Reihe von Problemen regelrecht überwältigt fühlen. Ob Krieg, Arbeitslosigkeit oder Kriminalität - die Lösungen für diese Probleme werden von der Politik verlangt. Wenn man an die begrenzten Eingriffsmöglichkeiten des Staates denkt, sind diese Forderungen nicht gerechtfertigt; aber sie zeigen den Fortbestand einer politischen Kultur an, die weiterhin am Primat der Politik festhält. Das läßt sich auch in anderer Hinsicht zeigen.

Wenn sich Politik auf die Organisation von Kontingenz beschränkt, wird die Zukunft nicht nur unkalkulierbar, sondern es entfällt auch der Bezugspunkt für eine bessere Zukunft, der den gegenwärtigen Entbehrungen ihren 
Sinn gibt. Eine derart degenerierte Politik erzeugt Qualen, da sie die ganze Last der Unsicherheit dem Individuum auflädt. Isoliert, ohne Bezugspunkte und soziale Sicherheit muß das Individuum alle Risiken selbst tragen.

Aufgrund ihrer Erfahrung von Hilflosigkeit und Unsicherheit können die Menschen Politik nur noch als eine selbstreferentielle Aktivität wahrnehmen. Auch wenn Politik vielleicht die materiellen Bedürfnisse der Menschen berïcksichtigt, so erzeugt sie doch nicht den grundlegenden und allgemein akzeptierten "Sinn für eine gesellschaftliche Ordnung", der als Maßstab und Referenzpunkt dienen könnte. Die Differenz zwischen Einzel- und Allgemeininteressen, zwischen Herrschenden und Beherrschten mag zwar unvermeidlich sein, doch die Kluft wird noch besonders akzentuiert, wenn Politik nicht länger als eine Sphäre der Repräsentation der Gesamtgesellschaft gilt. Auf der Seite der Institutionen schwindet die Verantwortung für die Probleme und wird diffus, was Politik überhaupt noch zu verantworten hat; auf der Seite der Bürger werden Verbindungen zur Gemeinschaft geschwächt und die öffentlichen Angelegenheiten erscheinen ihnen fremd und gleichgültig.

Die verschiedenen Symptome des Unbehagens - Mißtrauen, Unsicherheit, Gefühle der Vereinzelung, die verschwindende Identifikation mit politischen Parteien - signalisieren Mängel, die, wenn man sie umkehrt, jene kollektive Vorstellung umreißen, wie Politik sein sollte. Ich bin davon überzeugt, daß für einen großen Teil der derzeitigen sozialen Unruhen in Lateinamerika die offensichtlichen materiellen Forderungen nur der Funke sind. In Wirklichkeit geht es auch um die Forderung nach einer »guten Regierung « und einer wirklichen »Gemeinschaft von Bürgern«. Aber wie soll diese erstrebte Ordnung aussehen?

Eine politische Kultur kann zwar ein gewisses Maß an Beständigkeit erlangen, sie ist aber niemals völlig fixiert. Sie muß beständig reproduziert werden, indem immer wieder derjenige Kontext hergestellt wird, der darüber entscheidet, was als »normal und natürlich« gilt. Heute ist es, aufgrund der großen Veränderungen, die im Gange sind, schwierig geworden, die Symbole, Rituale, Gefühle und Werte zu reproduzieren, die die Demokratie, so wie wir sie kennen, ausmachen. Ein Merkmal unserer Epoche ist die Geschwindigkeit, mit der Erfahrungen veralten und zur Bewältigung der aktuellen Herausforderungen nicht mehr benutzt werden können. Dies gilt auch für unsere politischen Konzepte. Die Krise der ideologischen Koordinatensysteme, die seit den 20er Jahren das politische Panorama strukturierten, ist offensichtlich. Die Auflösung dieser Koordinatensysteme erweitert den Spielraum des »Möglichen«, allerdings nur um den Preis der Schwächung des Horizonts dessen »was wünschenswert ist«. Wir haben heute Schwierigkeiten, die angestrebten Ziele genauer zu umreißen und deshalb erscheint Politik als eine willkürliche Angelegenheit. 
Der Mangel an Interesse für Politik kann in allen Ländern beobachtet werden. Er sollte aber nicht mit Entpolitisierung verwechselt werden. Stattdessen scheint eine historische Perspektive sinnvoll zu sein, die im vorherrschenden Unbehagen jene neu entstandenen Orientierungen untersucht, die - ähnlich wie geologische Schichten - alte Strukturen überlagern. Das Politische verschwindet nicht aus der alltäglichen Erfahrung der Menschen, es erneuert sich im Einklang mit der neuen Marktgesellschaft, es wird gleichzeitig transnational und segmentiert. Da es sich hier um ein kulturelles Phänomen handelt, muß auch das neue kulturelle Klima berücksichtigt werden, wie es sich teilweise in der Debatte um die »Postmoderne« ausdrückt und erheblichen Einfluß auf die Idee der Demokratie ausübt (Lechner 1990).

Die vorangegangene Analyse erlaubt uns, einige Elemente sichtbar zu machen, die für die Transformation des Politischen eine Rolle spielen. Dabei handelt es sich weniger um neue Elemente, sondern um eine Umdeutung von bestehenden Ansprüchen, die auf eine neue »Bedeutung« von Demokratie verweisen.

Zunächst einmal ist es wichtig zu betonen, daß in allen lateinamerikanischen Ländern die Forderungen der Menschen, als Bürger anerkannt zu werden, öffentliche Resonanz erlangt haben. Im Gegensatz zur Anomymität und Atomisierung des Marktes verlangen die Bürger Anerkennung. Sie wollen als Subjekte anerkannt und in eine kollektive Ordnung integriert werden. Dies setzt einerseits rechtliche Anerkennung, also das »Recht Rechte zu haben « (Hannah Arendt), voraus. Wenn die Marktgesellschaft traditionelle Hierarchien nicht zerstört, sondern verstärkt - wie das in vielen Ländern der Region geschieht -, dann nimmt die »klassische « Forderung nach Sicherheit und Schutz die Form eines Aufschreis nach Gerechtigkeit und Rechtsstaatlichkeit an.

Zweitens implizieren die Forderungen nach Bürgerrechten eine Reform des politischen Systems und des Staates zu Einrichtungen, in denen sich die Bürger als »Bürgergemeinschaft « wiedererkennen können. Angesichts der stattfindenden Modernisierungsprozesse haben die bestehenden Institutionen keinen ausreichenden symbolischen Gehalt. Als Folge sozialer Differenzierung und Auffächerung bilden sich auf der Grundlage kollektiver Identitäten »Stämme« (Maffesoli), die untereinander nur wenig kommunizieren. Die rituelle Beschwörung von Solidarität nutzt hier wenig; es werden instrumentelle oder ökonomische Umgangsweisen notwendig, die mit der Brüderlichkeit, die gemeinsam mit Freiheit und Gleichheit die dritte Säule des republikanischen Lebens bildete, wenig zu tun haben. Die Unsicherheit der Marktgesellschaft wird durch die Auffächerung der kulturellen Formen und Gegebenheiten noch verschlimmert. In Lateinamerika gefähr- 
det der voranschreitende Säkularisierungsprozess auch jenen gemeinsamen Raum von Werten und Überzeugungen, Normen und Gewohnheiten, der mit allen notwendigen Einschränkungen - zu einer bestimmten »nationalen Kultur« beiträgt. Je weiter ein solcher Rahmen geschwächt wird, und in den meisten Gesellschaften der Region ist er schon sehr zerbrechlich, desto unsicherer werden auch die Gefühle der Zugehörigkeit und Identifikation mit der demokratischen Ordnung. Daher entstehen die Träume von der Konservierung oder Wiederherstellung früherer Formen der Gemeinschaft. Solche populistischen und fundamentalistischen Regressionen können nur vermieden werden, wenn die Demokratie selbst die Forderungen nach Gemeinschaft in ihre Obhut nimmt (Lechner 1991).

Damit wird die vorherrschende Konzeption von Demokratie, die sich auf Prozeduren und Institutionen des politischen Regimes bezieht, verändert, insofern nämlich Subjektivität an Bedeutung gewinnt. Die Stärkung der demokratischen Ordnung beinhaltet auch die Wiederaneignung der früher als irrational ausgeklammerten subjektiven Dimension (Hirschman 1980), um damit der Transformation des Politischen Rechnung zu tragen. Gerade um eine rationale Politik zu verteidigen, erscheint es mir notwendig, zu einer Rationalität zu finden, die der Komplexität der sozialen Realität angemessen ist.

Zuletzt möchte ich noch das gesteigerte Verlangen der Bürger nach politischer Führung hervorheben. Wie wir gesehen haben, werden durch die Schwerkraft der Marktgesellschaft die Steuerungskapazitäten reduziert und als Ausgleich die Koordinationsmechanismen gestärkt. Es ist richtig, daß eine bessere Koordination zwischen politischen und sozialen Akteuren, sowie zwischen Exekutivorganen, Parlament und Parteien, und vor allem auch innerhalb der Regierung, die Voraussetzung dafür ist, der Politik Kontinuität zu verleihen und zu vermeiden, daß das Pendel in Lateinamerika so häufig ausschlägt. Dennoch ist Kontinuität in der Politik nur möglich, wenn sie auf ein »nationales Projekt«, auf eine gemeinsame Vision eines wünschenswerten Zustandes abzielt. Ohne langfristige Ziele (wie im Fall des europäischen Vereinigungsprozesses der westeuropäischen Länder) geht die Perspektive, die es möglich macht, einzelne Initiativen zu artikulieren, verloren und Politik hängt lediglich von kontingenten Elemente ab. Politik verliert gerade ihr Spezifisches und wird mit den Konjunkturen der kapitalistischen Marktwirtschaft verwechselt. Das Gewicht der »allgegenwärtigen Gegenwart« ist aber gerade der Grund, warum die Menschen von der Politik eine verstärkte Steuerung der sozialen Prozesse erwarten.

Manchmal wird diese Zukunftsperspektive von einer charismatischen Autorität verkörpert. Dennoch wäre es ein Fehler, die Sehnsucht nach Führung mit der Sehnsucht nach einer solchen Führungspersönlichkeit zu ver- 
wechseln. Der gegenwärtige Mangel an Orientierung hat seine Wurzeln im Verlust eines gemeinsamen Rahmens; die Rekonstruktion eines solchen Rahmens ist die Voraussetzung dafür, daß Politik ihre Fähigkeit zurückgewinnt, die soziale Entwicklung zu steuern. Nur die Verbindung der Subjekte ermöglicht eine gemeinsame Konstruktion der Zukunft: einer Zunkunft als Verantwortung von uns allen. Wenn dies der Fall wäre, würde die Transformation des Politischen und der institutionalisierten Politik letztlich zur Verstärkung neuer Interpretationsmuster führen. Natürlich ist dies keine ausschließliche Aufgabe der Politik. Aber der dargestellte Zusammenhang unterstreicht die entscheidende Rolle, die eine so verstandene politische Kultur für die Erneuerung der Demokratie innerhalb des neuen Kontextes, und insgesamt für die Modernität Lateinamerikas, spielt.

\section{Übersetzung aus dem Englischen von Volker Lorek}

\section{Literature}

Altvater, Elmar (1991): Die Zukanft des Marktes, Münster.

BID - PNUD (1993): Reforna social y pobreza, BID.

Calderón, Fernando; Dos Santos, Mario (1991): Hacia un nuevo orden estatal en América Latina. Veinte tesis sociopolíticas y un corolario, Fondo de Cultura Económica, Mexiko.

Castoriadis, Cornelius (1994): La democracia ateniense y sus interpretaciones in: Vuelta 209, April.

Cavarozzi, Marcelo (1992): Beyond Transitions to Democracy in Latin America in: Journal of Latin American Studies 24.

CEPAL (1990): Transformación productiva con equidad, Santiago de Chile.

Held, David (1991): Democracy, the Nation State and the Global System in: Economy and Society.

Hirschman, Albert (1980): Leidenschafien und Interessen, Frankfurt/M.

Larraín, Felipe (1993): Los desafíos económicos del desarrollo político in: Drake, P.; Jaksic, I.: El dificil camino hacia la democracia en Chile 1982-1990, Santiago de Chile.

Lechner, Norbert (1990): Los patios interiores de la democracia. Subjetividad y políca, Fondo de Cultura Económica, Mexiko.

Lechner, Norbert (1991): The Search for Lost Community. Challenges to Democracy in Latin Amerika in: International Social Science Journal 129.

Maier, Charles (1987): Changing Boundaries of the Political, Cambridge.

Marin, Bernd; Mayntz, R.(1991): Polity Networks, New York. 